\title{
Reingeniería de las herramientas de gestión en los servicios y su incidencia con la calidad de atención de los usuarios del Hospital "Walter Cruz Vilca" Alto Moche, Trujillo
}

Reengineering of management tools in the services and its incidence with the quality of attention of the users of the "Walter Cruz Vilca" Hospital High Moche, Trujillo

$$
\begin{array}{r}
\text { Magna Rosa Trujillo Cuidad }{ }^{a} \text { escorpio 1965@ hotmail.com } \\
\text { ORCID: } \frac{\text { https://orcid.org/0000-0003-3587-3189 }^{\text {Universidad Nacional de Trujillo }}}{\text { Jorge Wilmer Elías Silupu }{ }^{b} \text { jelias s@ @ hotmail.es }} \\
\text { ORCID: } \frac{\underline{\text { https://orcid.org/0000-0002-0857-4258 }}}{\text { Universidad Nacional Ciro Alegría }} \\
\text { Carmen Rosa Cárdenas Rosales }{ }^{c} \text { crcardenasr@ unach.edu.pe } \\
\text { ORCID: } \frac{\text { https://orcid.org/0000-0001-7119-6002 }}{\text { Universidad Nacional Autónoma de Chota }}
\end{array}
$$

Recibido: Octubre / 05 /2021 - Revisado: Noviembre /25 / 2021 - Publicado: Diciembre /29 / 2021

\section{RESUMEN}

El objetivo del presente estudio fue determinar si la reingeniería de las herramientas de gestión en los servicios incide en la calidad de atención de los usuarios del Hospital "Walter Cruz Vilca” Alto Moche. Se llevó a cabo un estudio prospectivo, longitudinal, pre test-post test de tipo pre experimental en 296 usuarios externos a los cuales se les aplicó el SERVQUAL antes y después de la reingeniería de las herramientas gestión en el nosocomio mencionado. La calidad de atención de los usuarios del hospital "Walter Cruz Vilca” Alto Moche antes y después de la aplicación de la reingeniería a las herramientas de gestión en los servicios fue de 45,3 \% y 56,8 $\%$, con una mejora en la calidad de atención bajo a un 11,5\%. Se concluyó que la reingeniería a las herramientas de gestión en los servicios del Hospital Walter Cruz Vilca incide en la mejora en la calidad de atención percibida por el usuario externo en un 11,5\%.

El grado de conocimiento del personal administrativo sobre instrumentos de gestión en el Hospital Walter Cruz Vilca. Se evidencia que las frecuencias de categorización de grado regular (26,6\%) y malo (26,6\%) sumaron el 53,2 \% de ellos, muy aparte de ser los porcentajes más altos en este grupo. El porcentaje de grados de conocimiento restantes fueron: excelente (13,3\%), muy bueno (20\%) y bueno (13,3\%). Si bien el grado de conocimiento fue en su mayoría bajo, la presente evidencia generó, además como parte del estudio, que la directora del nosocomio y el equipo de gestión aunado al personal administrativo establezcan una reingeniería de las estrategias sanitarias y la implementación de planes de mejora y el correspondiente seguimiento y monitorización de los mismos, a fin de mejorar la calidad de atención percibida por el usuario externo.

Palabras claves: Reingeniería; herramientas de gestión; calidad de atención

\begin{abstract}
The objective of the present study was to determine if reengineering of the management tools in the services affects the quality of attention of the users of the Hospital "Walter Cruz Vilca" Alto Moche. A prospective, longitudinal, pre - test - post test was carried out in 296 external users to whom SERVQUAL was applied before and after the reengineering of
\end{abstract}


management tools in the mentioned hospital. The quality of attention of the users of the "Walter Cruz Vilca" Alto Moche hospital before and after the application of the reengineering to the tools of management in the services was $45.3 \%$ and $56.8 \%$, with an improvement in the Quality of care of $11.5 \%$. It was concluded that the reengineering to the management tools in the Walter Cruz Vilca Hospital services affects the improvement in the quality of care perceived by the external user in $11.5 \%$.

The degree of knowledge of the administrative staff on management instruments at the Walter Cruz Vilca Hospital. It was evidenced that the categorization frequencies of regular grade (26.6\%) and bad (26.6\%) totaled 53.2\% of them, well apart from being the highest percentages in this group. The percentage of degrees of knowledge remaining were excellent (13.3\%), very good (20\%) and good (13.3\%). Although the degree of knowledge was mostly low, the present evidence also generated, as part of the study, that the director of the hospital and the management team together with the administrative staff establish a reengineering of health strategies and the implementation of health plans. Improvement and the corresponding monitoring and monitoring thereof, in order to improve the quality of care perceived by the external user.

Keywords: Reengineering; management tools; attention quality

\section{Introducción}

En la actualidad han surgido diferentes filosofías y estrategias para la mejora de la eficiencia y la competitividad de las instituciones de salud. Una de éstas, es la reingeniería, que está basada en el rediseño radical de los procesos tanto a nivel operativo como administrativo para efectuar marcadas mejoras en medidas críticas y actuales de rendimiento, tales como costos, calidad, servicio y rapidez. (Herrera, 2008)

La Organización Panamericana de la Salud (OPS) ha informado que en América Latina uno de los principales desafíos del sector de salud es la de enfrentar en la actualidad y en las próximas décadas la reducción de la inequidad en el sistema sanitario en lo que concierne al acceso a los servicios y coberturas de salud. Esto ocurre porque el proceso de globalización de la economía, la reforma del estado, y en consecuencia la reforma del sector de la salud no ha conseguido responder a las demandas de la población en lograr mejores servicios de salud y mejor calidad de vida. (OPS, 2006).

La reingeniería en sistemas de salud generalmente está enfocada al apoyo de servicios directos a los usuarios, la logística, el abasto, las finanzas y el desarrollo de la investigación educación de los profesionales de la salud. Detrás de este escenario, las instituciones de salud 
están reconstruyendo las funciones de sus directivos para poder apoyar un desarrollo organizacional de mayor envergadura, con los esfuerzos de la reingeniería. Puntos clave de esa labor son la capacitación en sistemas modernos de gestión, la cultura organizacional de calidad y el liderazgo médico, encaminados a mejorar los procesos del primer contacto con los pacientes y a reducir significativamente los costos. La educación, la capacitación y la investigación deben estar sin duda en el centro de la reingeniería. (Revista Médica de salud, 2012).

Sin embargo, una de las estrategias utilizadas para reducir esa inequidad ha sido la búsqueda de una mejora de la calidad de los servicios, a partir de una definición del concepto de calidad, orientada a lograr tanto la satisfacción del usuario externo como la de la población, y la motivación del usuario interno que corresponde al trabajador del sector salud. También se ha intentado establecer la relación entre calidad, reducción de los costos y la equidad. (Herrera, 2008; OPS, 2006)

Los servicios de salud latinoamericanos han mantenido con el devenir de los años en diferentes etapas de crisis y deficientes servicios, insalubridad y falta de control en las instalaciones, falta de profesionalismo del personal médico y de enfermería, que se deben corregir imperantemente en pro del bienestar de nuestra sociedad al mejorar los servicios de atención médica. (Maceira, 2014)

Los gobiernos latinoamericanos preocupados del insuficiente sistema de servicios de las instituciones de salud promocionan la sacrificada tarea que se está realizando en los hospitales y centros de salud estatales, y, desarrollan gestiones de modernidad e implementación de nuevos sistemas con tecnología de punta para perfeccionar los materiales, recursos y el servicio de atención a los pacientes de la comunidad. (Atun et al, 2015)

Además de la problemática referida, los nosocomios públicos y privados en las últimas décadas han estado inmersos en un proceso continuo de adaptación, fruto de fuertes presiones sociales y políticas, cuyo objetivo es mejorar los niveles de calidad asistencial y reducir los costos operativos. Parece ser ésta la descripción de una situación generalizada 
del sector si atendemos al contenido de diversas publicaciones relacionadas directa o indirectamente con la gestión en el ámbito sanitario. (Padula, 2008)

Los expertos sanitarios han planteado que se adopten nuevas técnicas de gestión en los nosocomios, obligados de alguna manera por la política gubernamental que les plantea elevar sus niveles de asistencia para asi captar más pacientes - clientes y, por consiguiente, generar mayores recursos financieros en función de los servicios prestados. (Aparicio \& Triadó, 2007)

Por lo general cuando los nosocomios públicos o privados presentan falencias internas, a pesar de que estas no son percibidas por los clientes, ello perturba la operacionalidad de los establecimientos, generando disfuncionalidades que podrían motivar una gran dificultad organizacional dentro de esta. El resultado ha sido la adopción de diferentes técnicas y métodos de dirección, cuyo éxito en el sector empresarial ha inducido a prever idénticos resultados en el sector sanitario. (Giedion, Villar \& Ávila, 2010; Herrera, 2008)

\section{Material y métodos}

\section{Objeto de experimental:}

Diseño pre experimental.

$$
\mathrm{M}: \mathrm{O} 1 \quad \mathrm{X} \quad \mathrm{O} 2
$$

Donde:

M: muestra probabilística

O1: Pre tes aplicado a los usuarios

O2: Pos tes aplicado a los usuarios

$\mathrm{X}$ : Reingeniería de las herramientas de logística.

\section{Población:}

La población estuvo conformada por 3150 usuarios externos que acudieron en promedio mensual al hospital Walter Cruz Vilca - Alto Moche para cualquiera de las atenciones en los diferentes consultorios durante el periodo 2018. Además, se encuestó a 15 usuarios internos constituidos por personal administrativo. 


\section{Criterios de Selección}

a) Criterios de Selección:

- Pacientes mayores de 18 años que acuden para su atención al Hospital Walter Cruz Vilca.

- Pacientes que acepten participar en el estudio dando evidencia de ello mediante la firma del consentimiento informado.

\section{b) Criterios de Exclusión:}

- Pacientes se nieguen a participar en el estudio o que durante el desarrollo de este desistan de continuar en él.

- Pacientes con trastornos o enfermedades mentales.

\section{Muestra:}

La muestra se obtuvo 296 considerando al usuario externo de los consultorios del hospital que satisficieron con los requerimientos de selección. Se hizo uso del muestreo de tipo aleatorio sistemático considerando cada tres pacientes que ingresen al referido consultorio en los turnos de mañana y tarde.

\section{Métodos y técnicas}

La presente investigación usó metodología cuantitativa, longitudinal, descriptiva, aplicada.

Una vez aprobado el presente proyecto de investigación por la Universidad Nacional de Trujillo se realizaron las respectivas coordinaciones con las autoridades del hospital Walter Cruz Vilca de Alto Moche, para obtener el permiso necesario para la ejecución de la investigación.

Con dicha aceptación se entrevistaron a 296 usuarios externos y 15 usuarios internos que acudieron a dicho hospital para ser atendidos por cualquier servicio de atención.

Seleccionado el usuario se le explicó el propósito de la investigación solicitándole su consentimiento oral y escrito para formar parte de este estudio, ante la negación a 
participación se procedió a seleccionar de manera aleatoria a otro usuario por el contrario ante dicha aceptación se le entregó un cuestionario que tuvo que responder en un tiempo de 20 minutos.

Luego de conocidos los resultados de los cuestionarios, la directora del Hospital Walter Cruz Vilca diseñó un programa de mejoras dentro de un proceso una reingeniería a las herramientas de gestión en los servicios de salud nosocomiales.

Se reevaluó al año de su aplicación mediante el SERVQUAL a 296 usuarios externos a fin de conocer el impacto a través del resultado porcentual.

\section{Instrumentación}

- Cuestionario SERVQUAL: aplicado a los usuarios externos (pacientes).

- Instrumentos de Gestión Walter Cruz Vilca: aplicado a los usuarios internos (personal administrativo: directora de hospital, jefes de servicio, administrador, equipo de gestión).

\section{Análisis de resultados}

El procedimiento y análisis de datos se llevará a cabo con el uso de la informática para lo cual se creará una base de datos en SPSS. La estadística utilizada fue el análisis de frecuencias (porcentajes) en la evaluación pre y post de los 296 usuarios externos.

\section{Aspectos éticos}

- El vigente tratado se realizó respetando la declaración de Helsinsky II sobre las recomendaciones que guían a los medios en investigación biomédica que involucra a los seres humanos, se obtendrá también la aprobación del Comité de Investigación de la Escuela de Posgrado de la Universidad Nacional de Trujillo y del Hospital Walter Cruz Vilca. 
- Debido a que esta investigación se consideró sin riesgo y en cumplimiento con los aspectos mencionados en ella, se desarrolló por su diseño mediante encuesta por lo cual se contó con la aprobación de los participantes y requirió de consentimiento informado de los usuarios seleccionados.

- Toda la información proporcionada fue de carácter confidencial y sólo tuvo acceso a ella el personal investigador.

\section{$\underline{\text { Resultados }}$}

\section{Tabla 1:}

Calidad de atención de los usuarios externos según dimensiones antes de la intervención. Hospital Walter Cruz Vilca.

\begin{tabular}{ccccc}
\hline \multirow{2}{*}{ DIMENSIÓN } & \multicolumn{2}{c}{ SATISFACCIÓN } & \multicolumn{2}{c}{ INSATISFACCIÓN } \\
\cline { 2 - 5 } & Frecuencia & Porcentaje & Frecuencia & Porcentaje \\
\hline Seguridad & 152 & 51.4 & 144 & 48.6 \\
Empatía & 147 & 49.7 & 149 & 50.3 \\
Capacidad de Respuesta & 139 & 47.0 & 157 & 53.0 \\
Aspectos Tangibles & 123 & 41.6 & 173 & 58.4 \\
Fiabilidad & 110 & 37.2 & 186 & 62.8 \\
\hline TOTAL & 134 & $\mathbf{4 5 . 3}$ & 162 & 54.7 \\
\hline
\end{tabular}

FUENTE: Cuestionario SERVQUAL

La Tabla 1 nos muestra la calidad de atención de los usuarios externos según dimensiones antes de la intervención en el Hospital Walter Cruz Vilca 2018. La satisfacción general del usuario externo fue del 45,3 \%, siendo la dimensión de seguridad la que superó el 51,4 \%. Los porcentajes de satisfacción para las otras dimensiones fueron: empatía (49,7 $\%$ ), capacidad de respuesta (47\%), aspectos tangibles $(41,6 \%)$ y fiabilidad $(37,2 \%)$. Esto ocurre porque el proceso de globalización de la economía, la reforma del estado, y en consecuencia la reforma del sector de la salud no ha conseguido responder a las demandas de la población en lograr mejores servicios de salud y mejor calidad de vida. (OPS, 2006)

En contraste a nuestros resultados, Timana (2006), halló que el $48 \%$ de usuarios percibe la calidad de atención ofrecida en el servicio de emergencia de un nosocomio como 
de buena calidad, mientras el $45 \%$ la percibe como de regular calidad, es decir, menos de la mitad de los usuarios que acudieron al servicio de emergencia consideran la atención recibida como buena. En la dimensión estructura los usuarios la percibieron como de regular calidad con tendencia a ser de mala calidad. En la dimensión de procesos (percepción de la calidez del servicio), para el $57 \%$ de usuarios, la calidez fue regular; mientras un $37 \%$ refirió que es de buena calidad. La dimensión de resultados de la atención el 80 \% de usuarios consideró que sí mejoró su estado de salud después de la atención recibida. Concluyó que la percepción de la calidad de atención ofrecida en el servicio de emergencia fue regular en general y para cada una de las dimensiones.

El instrumento utilizado para nuestra investigación fue el SERVQUAL el cual es un instrumento válido y confiable en la evaluación de la calidad de servicio midiendo lo que el paciente espera de una institución prestadora de servicios de salud en las cinco dimensiones citadas, determinando así la discrepancia entre lo que el cliente espera del servicio y lo que percibe del mismo. Ello sirve de base como fuente de información para facilitar la puesta en marcha de medidas de corrección adecuadas que mejoren la calidad. (Chura, 2003).

\section{Figura 1.}

Porcentaje de calidad de atención de los usuarios externos según dimensiones antes de la intervención, hospital Walter Cruz Vilca. 2018.

Calidad de atenciòn de los usuarios externos

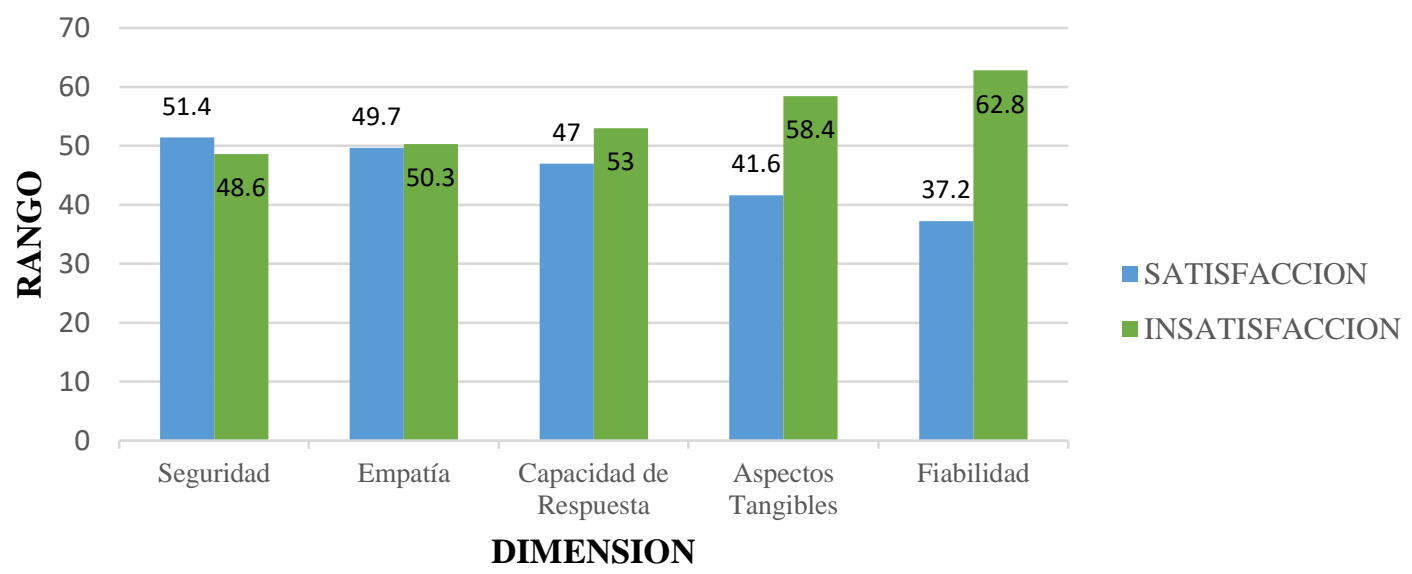

Fuente: Cuestionario Instrumentos de Gestión en el Hospital Walter Cruz Vilca 


\section{Tabla 2.}

Grado de conocimiento del personal administrativo sobre instrumentos de gestión en el Hospital Walter Cruz Vilca 2018.

\begin{tabular}{ccc}
\hline GRADO DE & \multicolumn{2}{c}{ PERSONAL ADMINISTRATIVO } \\
\cline { 2 - 3 } CONOCIMIENTO & Frecuencia & Porcentaje \\
\hline Excelente & 2 & $13.33 \%$ \\
\hline Muy bueno & 3 & $20.0 \%$ \\
\hline Bueno & 2 & $13,33 \%$ \\
\hline Regular & 4 & $26.6 \%$ \\
\hline Malo & 4 & $26.6 \%$ \\
\hline Total & $\mathbf{1 5}$ & $\mathbf{1 0 0 . 0} \%$
\end{tabular}

Fuente: Cuestionario Instrumentos de Gestión en el Hospital Walter Cruz Vilca

La Tabla 2 revela el grado de conocimiento del personal administrativo sobre instrumentos de gestión en el Hospital Walter Cruz Vilca. Se evidencia que las frecuencias de categorización de grado regular (26,6 \%) y malo (26,6 \%) sumaron el 53,2 \% de ellos, muy aparte de ser los porcentajes más altos en este grupo. El porcentaje de grados de conocimiento restantes fueron: excelente $(13,3 \%)$, muy bueno $(20 \%)$ y bueno $(13,3 \%)$. Si bien el grado de conocimiento fue en su mayoría bajo, la presente evidencia generó, además como parte del estudio, que la directora del nosocomio y el equipo de gestión aunado al personal administrativo establezcan una reingeniería de las estrategias sanitarias y la implementación de planes de mejora y el correspondiente seguimiento y monitorización de estos, a fin de mejorar la calidad de atención percibida por el usuario externo. La reingeniería está basada en el rediseño radical de los procesos tanto a nivel operativo como administrativo para efectuar marcadas mejoras en medidas críticas y actuales de rendimiento tales como costos, calidad, servicio y rapidez. (Herrera, 2008) 


\section{Figura 2:}

Porcentaje del grado de conocimiento del personal administrativo sobre instrumentos de gestión en el Hospital Walter Cruz Vilca 2018.

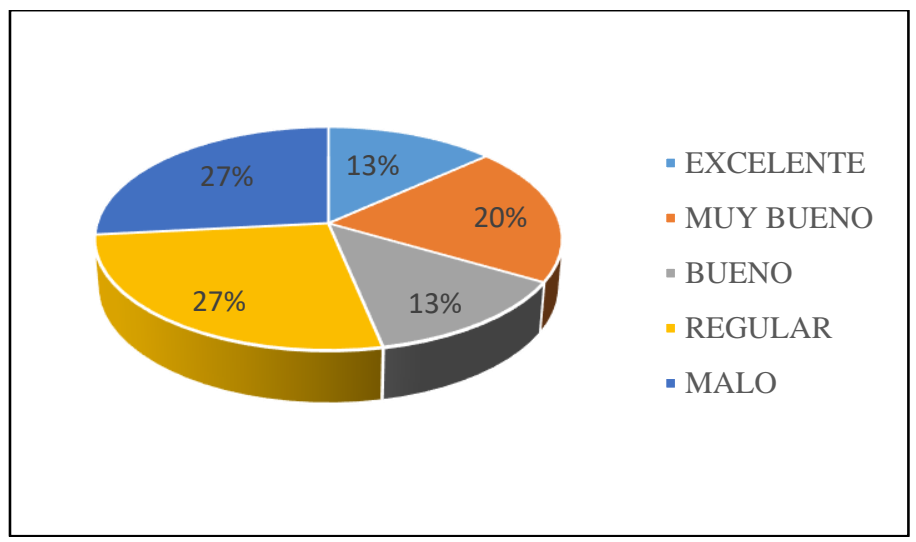

Fuente: Cuestionario SERVQUAL

\section{Tabla 3:}

Calidad de atención de los usuarios externos según dimensiones post intervención. Hospital Walter Cruz Vilca 2018

\begin{tabular}{ccccc}
\hline \multirow{2}{*}{ DIMENSIÓN } & \multicolumn{2}{c}{ SATISFACCIÓN } & \multicolumn{2}{c}{ INSATISFACCIÓN } \\
\cline { 2 - 5 } & Frecuencia & Porcentaje & Frecuencia & Porcentaje \\
\hline Fiabilidad & 193 & 65.2 & 103 & 34.8 \\
\hline Seguridad & 185 & 62.5 & 111 & 37.5 \\
\hline Empatía & 170 & 57.4 & 126 & 42.6 \\
\hline Capacidad de Respuesta & 159 & 53.7 & 137 & 46.3 \\
\hline Aspectos Tangibles & 151 & 51.0 & 145 & 49.0 \\
\hline TOTAL & 168 & 56.8 & 128 & 43.2 \\
\hline
\end{tabular}

La Tabla 3 muestra la calidad de atención de los usuarios externos según dimensiones post intervención en el Hospital Walter Cruz Vilca en agosto del 2018. La satisfacción general del usuario externo fue del $56,8 \%$, siendo la dimensión de fiabilidad la de mayor registro porcentual $(65,2 \%)$, seguida de las dimensiones seguridad (62,5\%), empatía (57,4 $\%)$, capacidad de respuesta $(53,7 \%)$ y aspectos tangibles $(51 \%)$. 
El modelo SERVQUAL de la calidad de servicio está basado en un enfoque de evaluación del cliente sobre la calidad de servicio en el que define un servicio de calidad como la diferencia entre las expectativas y percepciones de los clientes. De este modo, un balance ventajoso para las percepciones, de manera que éstas superaran a las expectativas, implicaría una elevada calidad percibida del servicio, y alta satisfacción con el mismo. SERVQUAL señala ciertos factores clave que condicionan las expectativas de los usuarios: comunicación "boca a boca", u opiniones y recomendaciones de amigos y familiares sobre el servicio, necesidades personales, experiencias con el servicio que el usuario haya tenido previamente, y, comunicaciones externas, que la propia institución realice sobre las prestaciones de su servicio y que incidan en las expectativas que el ciudadano tiene sobre las mismas. (Chávez, 2002; Chura, 2003)

\section{Figura 3:}

Porcentaje de Calidad de atención de los usuarios externos según dimensiones post intervención. Hospital Walter Cruz Vilca. 2018.

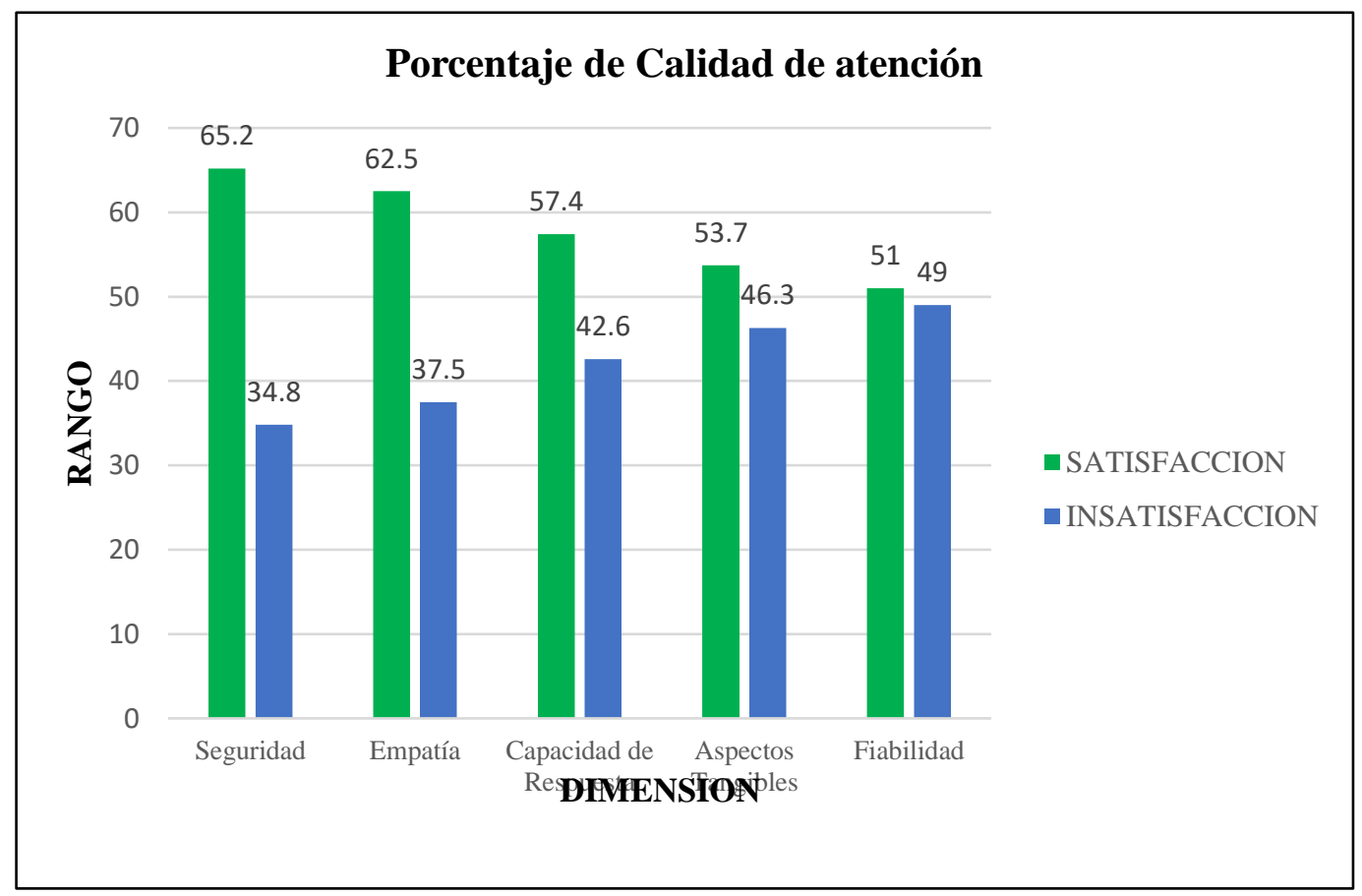

Fuente: Cuestionario SERVQUAL 


\section{Tabla 4:}

Comparación de los cambios en la satisfacción de los usuarios externos según dimensiones pre y post intervención. Hospital Walter Cruz Vilca. 2018.

\begin{tabular}{ccccccc}
\hline DIMENSIÓN & \multicolumn{2}{c}{$\begin{array}{c}\text { SATISFACCIÓN } \\
\text { ANTES }\end{array}$} & \multicolumn{2}{c}{$\begin{array}{c}\text { SATISFACCIÓN } \\
\text { DESPUES }\end{array}$} & \multicolumn{2}{c}{ VARIACIÓN } \\
\cline { 2 - 7 } & Frecuencia & Porcentaje & Frecuencia & Porcentaje & Frecuencia & Porcentaje \\
\hline Fiabilidad & 110 & 37.2 & 193 & 65.2 & 83 & 28.0 \\
\hline $\begin{array}{c}\text { Aspectos } \\
\text { tangibles }\end{array}$ & 123 & 41.6 & 151 & 51.0 & 28 & 9.5 \\
\hline Empatía & 147 & 49.7 & 170 & 57.4 & 23 & 7.8 \\
\hline $\begin{array}{c}\text { Capacidad de } \\
\text { respuesta }\end{array}$ & 139 & 47.0 & 159 & 53.7 & 20 & 6.8 \\
\hline Seguridad & 187 & 63.2 & 185 & 62.5 & -2 & -0.7 \\
\hline TOTAL & $\mathbf{1 3 4}$ & $\mathbf{4 5 . 3}$ & $\mathbf{1 6 8}$ & $\mathbf{5 6 . 8}$ & $\mathbf{3 4}$ & $\mathbf{1 1 . 5}$ \\
\hline & & Fuente: Cuestionario SERVQUAL & &
\end{tabular}

La Tabla 4 nos proporciona información sobre la comparación de los cambios en la satisfacción de los usuarios externos según dimensiones pre y post intervención (reingeniería) en el sistema funcional administrativo y asistencial del Hospital Walter Cruz Vilca de agosto 2017 a agosto 2018. Se observó una marcada mejora porcentual general de la calidad de atención que percibe el usuario externo con respecto a la atención recibida, en agosto del 2015 fue de 45,3 \% y en agosto del 2016, 56,8 \%. De igual forma para las dimensiones que la componen, excepto en la dimensión seguridad en la que hubo una disminución del 0,7\%.

SERVQUAL posee cinco dimensiones relativas a los criterios de evaluación que utilizan los clientes para valorar la calidad en un servicio y son definidas, como ya se ha expuesto, del siguiente modo: Fiabilidad (habilidad para realizar el servicio de modo cuidadoso y fiable), Capacidad de Respuesta (disposición y voluntad para ayudar a los usuarios y proporcionar un servicio rápido), Seguridad (conocimientos y atención mostrados por los empleados y sus habilidades para concitar credibilidad y confianza), Empatía (atención personalizada que dispensa la organización a sus clientes), y, Elementos Tangibles 
(apariencia de las instalaciones físicas, equipos, personal y materiales de comunicación). (Herrera, 2008; Chávez, 2002)

\section{Figura 4:}

Porcentaje de la variación de cuadro Comparación de los cambios en la satisfacción de los usuarios externos según dimensiones pre y post intervención. Hospital Walter Cruz Vilca. 2018 .

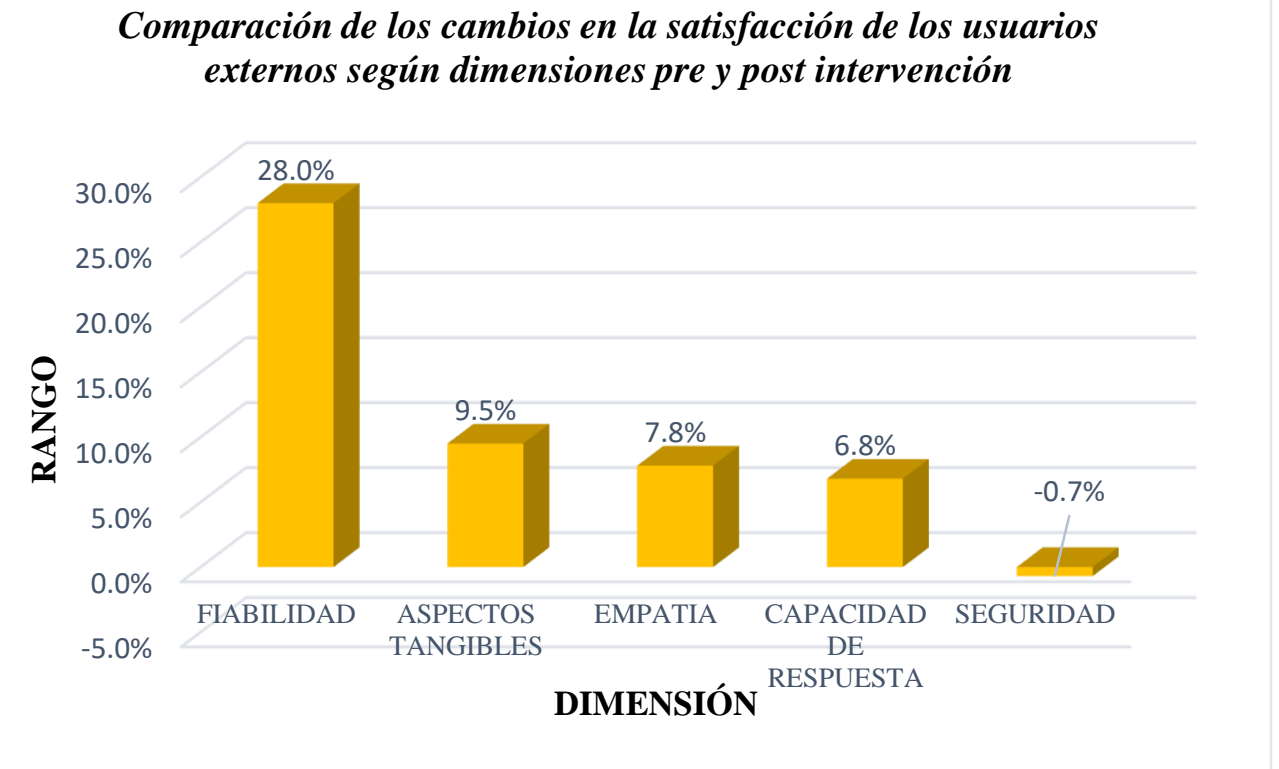

\section{Discusión}

La Tabla 1 nos muestra la calidad de atención de los usuarios externos según dimensiones antes de la intervención en el Hospital Walter Cruz Vilca 2018. La satisfacción general del usuario externo fue del 45,3 \%, siendo la dimensión de seguridad la que superó el 51,4\%. Los porcentajes de satisfacción para las otras dimensiones fueron: empatía (49,7 $\%)$, capacidad de respuesta (47\%), aspectos tangibles $(41,6 \%)$ y fiabilidad $(37,2 \%)$. Esto ocurre porque el proceso de globalización de la economía, la reforma del estado, y en consecuencia la reforma del sector de la salud, no ha conseguido responder a las demandas de la población en lograr mejores servicios de salud y mejor calidad de vida. (OPS, 2006). 
En contraste a nuestros resultados, Timana (2006), halló que el $48 \%$ de usuarios percibe la calidad de atención ofrecida en el servicio de emergencia de un nosocomio como de buena calidad, mientras el $45 \%$ la percibe como de regular calidad, es decir, menos de la mitad de los usuarios que acudieron al servicio de emergencia consideran la atención recibida como buena. En la dimensión estructura los usuarios la percibieron como de regular calidad con tendencia a ser de mala calidad. En la dimensión de procesos (percepción de la calidez del servicio), para el $57 \%$ de usuarios, la calidez fue regular; mientras un $37 \%$ refirió que es de buena calidad. La dimensión de resultados de la atención el 80 \% de usuarios consideró que sí mejoró su estado de salud después de la atención recibida. Concluyó que la percepción de la calidad de atención ofrecida en el servicio de emergencia fue regular en general y para cada una de las dimensiones.

El instrumento utilizado para nuestra investigación fue el SERVQUAL el cual es un instrumento válido y confiable en la valoración de la calidad de servicio midiendo lo que el paciente espera de una institución prestadora de servicios de salud en las cinco dimensiones citadas, determinando así la discrepancia entre lo que el cliente espera del servicio y lo que percibe del mismo. Ello sirve de base como fuente de información para facilitar la puesta en marcha de medidas de corrección adecuadas que mejoren la calidad. (Chura, 2003).

La Tabla 2 revela el grado de conocimiento del personal administrativo sobre instrumentos de gestión en el Hospital Walter Cruz Vilca. Se evidencia que las frecuencias de categorización de grado regular (26,6 \%) y malo (26,6\%) sumaron el 53,2 \% de ellos, muy aparte de ser los porcentajes más altos en este grupo. El porcentaje de grados de conocimiento restantes fueron: excelente $(13,3 \%)$, muy bueno $(20 \%)$ y bueno $(13,3 \%)$. Si bien el grado de conocimiento fue en su mayoría bajo, la presente evidencia generó, además como parte del estudio, que la directora del nosocomio y el equipo de gestión aunado al personal administrativo establezcan una reingeniería de las estrategias sanitarias y la implementación de planes de mejora y el correspondiente seguimiento y monitorización de los mismos, a fin de mejorar la calidad de atención percibida por el usuario externo. La reingeniería está basada en el rediseño radical de los procesos tanto a nivel operativo como 
administrativo para efectuar marcadas mejoras en medidas críticas y actuales de rendimiento tales como costos, calidad, servicio y rapidez. (Herrera, 2008).

La Tabla 3 muestra la calidad de atención de los usuarios externos según dimensiones post intervención en el Hospital Walter Cruz Vilca en agosto del 2018. La satisfacción general del usuario externo fue del 56,8 \%, siendo la dimensión de fiabilidad la de mayor registro porcentual $(65,2 \%)$, seguida de las dimensiones seguridad (62,5\%), empatía (57,4 $\%)$, capacidad de respuesta $(53,7 \%)$ y aspectos tangibles $(51 \%)$.

El modelo SERVQUAL de la calidad de servicio se basa en la evaluación que hace el cliente por el servicio que ha recibido y como es que este los percibe. Así si obtenemos un balance ventajoso en la percepción del servicio superaría las expectativas de los usuarios, teniendo una alta satisfacción por sus servicios. SERVQUAL tiene en cuenta ciertos factores como el de la comunicación "boca a boca", opiniones y recomendaciones de allegados, amigos y familiares, así como todas aquellas experiencias de servicios al que se han visto inmersos los usuarios y pacientes de tal forma que representen un impacto positivo en función de sus expectativas de servicio. (Chávez, 2002; Chura, 2003).

La Tabla 4, nos proporciona información sobre la comparación de los cambios en la satisfacción de los usuarios externos según dimensiones pre y post intervención (reingeniería) en el sistema funcional administrativo y asistencial del Hospital Walter Cruz Vilca de agosto 2017 a agosto 2018. Se observó una marcada mejora porcentual general de la calidad de atención que percibe el usuario externo con respecto a la atención recibida, en agosto del 2015 fue de 45,3 \% y en agosto del 2016, 56,8 \%. De igual forma para las dimensiones que la componen, excepto en la dimensión seguridad en la que hubo una disminución del 0,7\%.

SERVQUAL posee cinco dimensiones relativas a los criterios de evaluación que utilizan los clientes para valorar la calidad en un servicio y son definidas, como ya se ha expuesto, del siguiente modo: Fiabilidad (habilidad para realizar el servicio de modo cuidadoso y fiable), Capacidad de Respuesta (disposición y voluntad para ayudar a los usuarios y proporcionar un servicio rápido), Seguridad (conocimientos y atención mostrados 
por los empleados y sus habilidades para concitar credibilidad y confianza), Empatía (atención personalizada que dispensa la organización a sus clientes), y, Elementos Tangibles (apariencia de las instalaciones físicas, equipos, personal y materiales de comunicación). (Herrera, 2008; Chávez, 2002).

\section{$\underline{\text { Conclusiones }}$}

- La calidad de atención de los usuarios del hospital "Walter Cruz Vilca" Alto Moche antes de la aplicación de la reingeniería a las herramientas de gestión en los servicios fue de $45,3 \%$.

- La reingeniería a las herramientas de gestión en los servicios del Hospital Walter Cruz Vilca incide en la mejora en la calidad de atención percibida por el usuario externo en un $11,5 \%$.

- La calidad de atención de los usuarios del hospital "Walter Cruz Vilca" Alto Moche después de la aplicación de la reingeniería a las herramientas de gestión en los servicios fue de $56,8 \%$.

- El grado de conocimiento del personal administrativo sobre los instrumentos de gestión, se evidencia que las frecuencias de categorizaciones con grado regular con un $26,6 \%$ seguido de un 53,2 \%. Si bien el grado de conocimiento fue en su mayoría bajo, esta generara que los directores y equipos de gestión de dicho nosocomio establezcan una reingeniería de las estrategias sanitarias y la implementación de planes de mejora a fin de mejorar la calidad de atención percibida tanto por el usuario externo como interno.

\section{$\underline{\text { Referencias }}$}

Aparicio, M., Triadó, X. (2007). Diseño organizativo de los hospitales y estructuras flexibles, un reto asumible. Actas del XIV Congreso Nacional de la Asociación de Economía y Dirección de Empresas. España.

Armijo, A. (2012). Reingeniería a los procesos administrativos y contables de la Fundación Ecuatoriana de Estudios Ecológicos "ECOCIENCIA". Tesis para obtener el Título de 
Ingeniera en Contabilidad y Auditoría Contadora Pública Autorizada. Universidad Central del Ecuador. Quito, Ecuador.

Artaza, B., Castro, A., González, O., Kuhn, B., Madrid, S., Marconi, R., et al. (2011). Economía y salud: aportes y experiencias en América Latina. Chile: OMS/OPS.

Belenes, R. (2008). Innovaciones en la gestión: nueva cultura empresarial en los servicios sanitarios. En: Gestión de hospitales, nuevos instrumentos y tendencias. Barcelona: Editorial Vicens - Vives.

Chávez, M. (2002). Evaluación de la calidad del servicio, desde la percepción del usuario externo. Centro de Salud Augusto B. Leguía. Tacna. Tesis para optar el Título de Médico Cirujano. Universidad Peruana Cayetano Heredia. Lima, Perú.

Chura, F. (2003). Evaluación de la calidad del servicio desde la percepción del usuario externo Centro de Salud La Esperanza. Tesis para optar el Título de Médico Cirujano. Universidad Peruana Cayetano Heredia. Lima, Perú.

Disease Management Association of America. (s.f.). Disponible en http://www.dmaa.org: Disponible en http://www.dmaa.org

Donabedian, A. (1990). Garantía y calidad de la atención médica. México: Instituto Nacional de Salud Pública.

Donabedian, Avedis. (1995). Calidad de atención a la salud. México: Instituto Nacional de Salud Pública.

Gómez, D. (2005). Análisis comparado de los sistemas de salud de la Región Andina y El Caribe. Rev Salud Pública, 7(3), 305 - 16.

Gonzáles, M. (2002). La Percepción. México, D.F.: México.

Hammer, M. (1990). Reengineering Work: Don't automate, obliterate. Harvard Business Review, 7, $104-12$.

Hammer, M; Champy, J. (1993). Reengineering the corporation: a manifesto for businnes revolution. New York: Harper Collins Publishers.

Herrera, M. (2008). Reingeniería. Costa Rica: Editorial Cosmos.

Maceira, D. (2014). Cuadrantes de análisis en los sistemas de salud de América Latina. Buenos Aires: UNICEF.

Mancheno, C. (2014). Diseño de un modelo de gestión de procesos para el servicio de emergencia de la Clínica "Médicos y Asociados Clínchimborazo Cía. Ltda.", de la ciudad de Riobamba, año 2013. Tesis para obtener el Título de Magister en Gestión Empresarial. Universidad Técnica Particular de Loja. Loja, Ecuador. 
Melinkoff, R. (1990). Los procesos administrativos. Caracas: PANAPO.

Morales, M. Calidad de atención desde la perspectiva del paciente. Tesis para optar el Grado de Maestría en Ciencias de Enfermería con Énfasis en Administración de Servicios. Universidad Autónoma de Nuevo León. México D.F., México.

Murillo, C., Saurina, C. Medida de la importancia de las dimensiones de la satisfacción en la provisión de servicios de salud. Gac Sanit, 27(4), 304 - 9.

Organización Panamericana de la Salud, Área de Sistemas de Salud Basados en Atención Primaria de Salud, Proyecto de Servicios Integrados de Salud. (2010). Metodología de gestión productiva de los servicios de salud: introducción y generalidades. Washington, D.C.: OPS.

Padula, C. (2008). Reorganizar los hospitales. España: Papeles de Economía Española.

Paudel, D. Quality of antenatal care and institutional delivery practice in Nepal: Further analysis of 2011 Nepal Demographic and Health Survey. Ad Astra, 5(2), 29 - 33.

Quintana, J.M., Aróstegui, I., Arcelay, A., García, M., Ortega, M., Candelas, S. (1999). Encuesta de satisfacción a pacientes de servicios de urgencias de hospitales agudos. Gac Sanit, 13(1), 38 - 45.

Real Academia de la Lengua Española. (2001). Diccionario de la lengua española. Madrid: Espasa.

Rosales, E., Miranda, J., Lema, C., López, L., Paca, A., Luna, D., et al. (2011). Recursos y capacidades de servicios de emergencia para atención de lesiones por traumas en Perú. Cad. Saúde Pública, 27(9), 1837 - 46.

Talavera, C. (2013). Métodos y herramientas de mejora aplicados en la administración pública. Granada: Unión Iberoamericana de Municipalistas.

Timaná, L. (2006). Percepción de la Calidad de Atención por el usuario externo del servicio de Emergencia del Hospital Nacional Daniel Alcides Carrión; Lima 2002. Revista Portales Médicos. Disponible en: http://www.portalesmedicos.com/publicaciones/articles/24/1/Percepcion-de-lacalidad-de-atencion-por-el-usuario-externo-del-servicio-de-emergencia-delhospital-nacional-Daniel-Alcides-Carrion---Callao-Peru.html

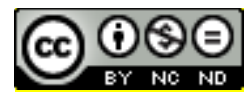

Reingeniería de las herramientas de gestión en los servicios y su incidencia con la calidad de atención de los usuarios del Hospital "Walter Cruz Vilca" Alto Moche, Trujillo (Magna Rosa Trujillo - Cuidad) Por Revista Sendas se encuentra bajo una Licencia Creative Commons-No Comercial-Sin Derivadas 3.0 Uported. 